\title{
Correction to: Consensus Definitions and Interpretation Templates for Fluoroscopic Imaging of Defecatory Pelvic Floor Disorders
}

\author{
Proceedings of the Consensus Meeting of the Pelvic Floor Consortium of the American \\ Society of Colon and Rectal Surgeons, the Society of Abdominal Radiology, the International \\ Continence Society, the American Urogynecologic Society, the International Urogynecological \\ Association, and the Society of Gynecologic Surgeons
}

\author{
Ian Paquette ${ }^{1} \cdot$ David Rosman $^{2} \cdot$ Rania El Sayed $^{3} \cdot$ Tracy Hull $^{4} \cdot$ Ervin Kocjancic $^{5} \cdot$ Lieschen Quiroz $^{6}$.

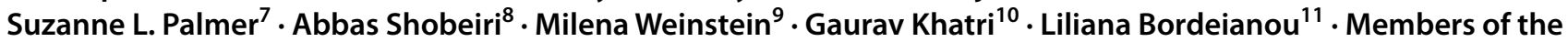 \\ Expert Workgroup on Fluoroscopic Imaging of Pelvic Floor Disorders
}

Published online: 28 January 2021

(c) The American Society of Colon and Rectal Surgeons and American Urogynecologic Society 2021

\section{Correction to: Techniques in Coloproctology https://doi.org/10.1007/s10151-020-02376-6}

In the article "Consensus definitions and interpretation templates for fluoroscopic imaging of defecatory pelvic floor disorders," which appeared in Volume 64, Issue 1 of Diseases of the Colon \& Rectum, the authors found an error in the spelling of the first name of author 7. For the record, the corrected first name is Suzanne L. Palmer, M.D., F.A.C.P.

The original article can be found online at https://doi.org/10.1007/ s10151-020-02376-6.

Liliana Bordeianou

lbordeianou@mgh.harvard.edu

1 Department Colorectal Surgery, University of Cincinnati, Cincinnati, $\mathrm{OH}$, USA

2 Department of Radiology, Pelvic Floor Disorders Center at the Massachusetts General Hospital, Harvard Medical School, Boston, MA, USA

3 Department of Radiology, Cairo University Pelvic Floor Centre of Excellency and Research Lab at Cairo University Faculty of Medicine and Teaching Hospitals, Cairo, Egypt

4 Department of Colorectal Surgery, Cleveland Clinic Hospitals, Cleveland, OH, USA

5 Department of Urology, University of Illinois, Chicago, IL, USA

6 Department of Obstetrics and Gynecology, University of Oklahoma, Oklahoma City, OK, USA

\section{Reference}

Paquette I, Rosman D, El Sayed R et al (2021) Consensus definitions and interpretation templates for fluoroscopic imaging of defecatory pelvic floor disorders. Dis Colon Rectum 64:31-44. https:// journals.lww.com/dcrjournal/Fulltext/2021/01000/Consensus_ Definitions_and_Interpretation_Templates.11.aspx

Publisher's Note Springer Nature remains neutral with regard to jurisdictional claims in published maps and institutional affiliations.
7 Department of Radiology, Keck Medical Center of USC, Los Angeles, CA, USA

8 Department of Obstetrics and Gynecology, University of Virginia, INOVA Women's Hospital, Falls Church, VA, USA

9 Department of Obstetrics a Gynecology, Massachusetts General Hospital Pelvic Floor Disorders Center, Harvard Medical School, Boston, MA, USA

10 Department of Radiology, UT Southwestern Medical Center, Dallas, TX, USA

11 Section of Colorectal Surgery, Division of General and Gastrointestinal Surgery, Massachusetts General Hospital Pelvic Floor Disorders Center, Harvard Medical School, 15 Parkman St, WACC 460, Boston, MA 02114, USA 